\title{
All-Optical Pulse Retiming Based on Quadratic Cascading in a Periodically Poled Lithium Niobate Waveguide
}

\author{
Kwang Jo Lee, Sheng Liu, Francesca Parmigiani, Periklis Petropoulos, David Richardson \\ Optoelectronics Research Centre, University of Southampton, Southampton, SO17 1BJ, UK \\ Katia Gallo \\ Department of Applied Physics, Royal Institute of Technology (KTH), 10691 Stockholm, Sweden
}

\begin{abstract}
We demonstrate an all-optical technique for the elimination of timing jitter in short pulse transmission systems. The technique relies on pulse pre-shaping followed by optical switching in a periodically poled lithium niobate waveguide via cascaded second harmonic and difference frequency generation.

(C)2010 Optical Society of America

OCIS codes: (060.2330) Fiber optics communications; (130.3730) Lithium niobate; (190.2620) Harmonic generation and mixing; (190.4410) Nonlinear optics, parametric processes; (230.4320) Nonlinear optical devices; (270.2500) Fluctuations, relaxations, and noise.
\end{abstract}

\section{Introduction}

The cumulative effects of repeater noise amplification and optical nonlinearities in long-haul transmission systems can cause either amplitude noise or timing jitter in optical signals, resulting in significant performance degradation. Therefore, signal regeneration techniques (or elimination of both amplitude noise and timing jitter) are required to overcome these transmission impairments. Various schemes to eliminate the timing jitter have been demonstrated previously, including schemes that either use a phase modulator synchronously driven at the data repetition rate [1], or that optically switch the data pulses with synchronous pulses of a rectangular temporal profile [2]. However, the scheme in [1] has a relatively low switching speed limited by the electrical bandwidth of the modulator/drive electronics, and the stability of the systems exemplified within [2], which are based on $\chi^{(3)}$ nonlinearities, is generally compromised by the long fiber lengths required for efficient operation. Such limitations can easily be overcome using their compact $\chi^{(2)}$ counterparts $[3,4]$.

In recent years, the use of cascaded second-order nonlinear processes in periodically poled lithium niobate (PPLN) waveguides has attracted considerable interest as a promising route to realize all-optical signal processing. The technology provides for high nonlinear coefficients, an ultra-fast optical response, bit rate and modulation format transparency, low spontaneous emission noise, low cross talk, and no intrinsic frequency chirp [5]. Cascaded second-harmonic and difference-frequency generation (cSHG/DFG) and cascaded sum- and difference-frequency generation (cSFG/DFG) have both been exploited in various all-optical signal processing applications [5].

In this paper, we demonstrate an all-optical technique for the elimination of timing jitter based on cSHG/DFG in a fiberized PPLN waveguide. The technique relies on pre-shaping RZ-signal data pulses (7 ps) into longer rectangular pulses $(50 \mathrm{ps})$ and then switching these pulses in a PPLN waveguide with a synchronous clean optical clock signal.

\section{Experiment and discussion}

Figure 1 illustrates the experimental setup used to realize our all-optical pulse retiming system based on cSHG/DFG in a PPLN waveguide. A 30-mm-long fiber pigtailed PPLN waveguide (HC Photonics Corp.) was used for the cSHG/ DFG process, and its SHG phase matching wavelength was $1546 \mathrm{~nm}$ at $50^{\circ} \mathrm{C}$. A $10-\mathrm{GHz}, 1.5-\mathrm{ps}$ modelocked erbium glass oscillator (ERGO) was filtered using a $0.5 \mathrm{~nm}$-bandpass filter (yielding a temporal width of 7 ps) to match the PPLN SHG acceptance bandwidth. The generated data pulses were modulated by a $2^{31}-1$ pseudorandom bit sequence (PRBS) using a lithium niobate modulator. These data pulses were amplified and launched into an optical processor (Finisar Waveshaper 4000E), which re-shaped them into 50 ps-long (FWHM) rectangular pulses as shown in Fig. 1(a). The pulses had sharp trailing and leading edges and a good flat-top section. The clean clock pulses at a wavelength of $1550 \mathrm{~nm}$ were generated by a 10-GHz, 7-ps gain-switched laser (GSL) [see Fig. 1(b)], and amplified before being combined with the rectangular pulses at a 3-dB coupler and launched into the PPLN waveguide. A variable delay line was used to produce a well defined delay between the clean clock and rectangular pulse trains and simulate timing jitter. The noisy input train of reshaped rectangular pulses at $1546 \mathrm{~nm}$ was up-converted to $773 \mathrm{~nm}$ by the SHG process in the PPLN waveguide. The SH data stream, which still retained the timing jitter and noise of the input, was then gated by the input clock via DFG to eliminate the jitter and produce 
a clean replica of the original data. Figure 1(d) shows the output spectrum of the PPLN waveguide monitored with an optical spectrum analyzer (OSA). The optical signal to noise ratio of the output signal was more than $22 \mathrm{~dB}$. A tunable bandpass filter (Alnair Labs.) was used after the PPLN device to extract the output signal. The measured eye-diagrams are also shown in Fig. 1(c).

The measured peak power and temporal position of the output pulses are plotted in Fig. 2 as a function of the temporal offset between the pulse streams. The zero on the horizontal axis was chosen to coincide with the point providing the most efficient conversion (or the maximum peak power). Figure 2(a) corresponds to the case in which the input TDM pulses have been reshaped into rectangular pulses, while Fig. 2(b) corresponds to the case where the device is directly driven by the input signal (7-ps FWHM Gaussian pulses as in the case of [4]). The curves in Fig. 2 clearly show an improvement of the retiming characteristics of the device associated with the preliminary reshaping of the input signal pulses. We also assessed the performance of the system through bit-error rate (BER) measurements. Error-free operation $\left(\mathrm{BER}=10^{-9}\right.$ ) was achieved and the power penalty was $0.6 \mathrm{~dB}$ as compared to the back-to-back measurements (at $10 \mathrm{Gbit} / \mathrm{s}$ ).

$0.5 \mathrm{~nm} \mathrm{BPF} \quad$ Optical processor

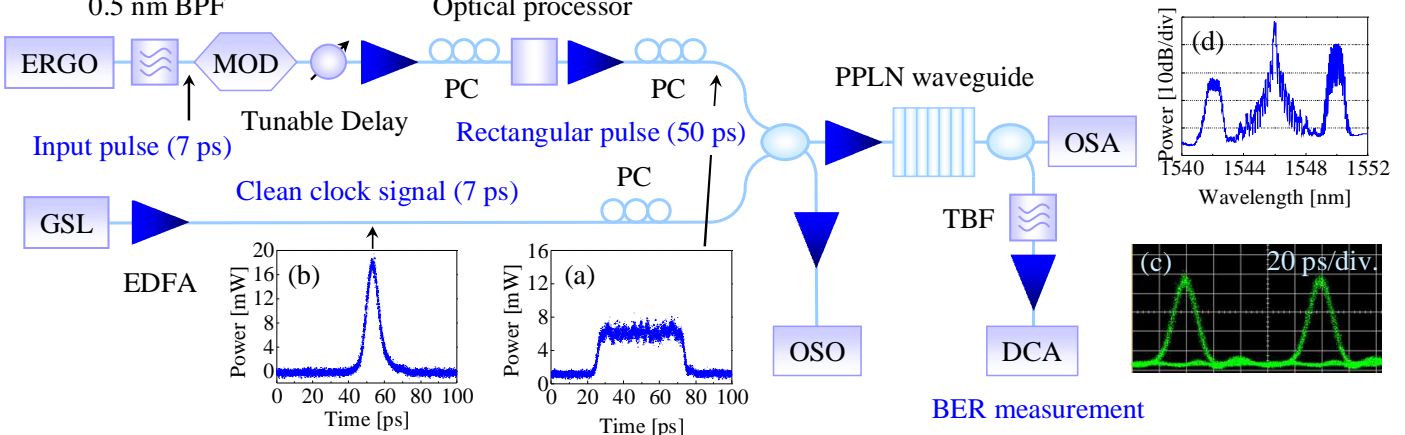

Fig. 1. Schematic of the experimental setup: MOD: modulator, EDFA: erbium-doped fiber amplifier, PC: polarization controller, DCA: digital communication analyzer, OSO: optical sampling oscilloscope, TBF: tunable bandpass filter, OSA: optical spectrum analyzer.

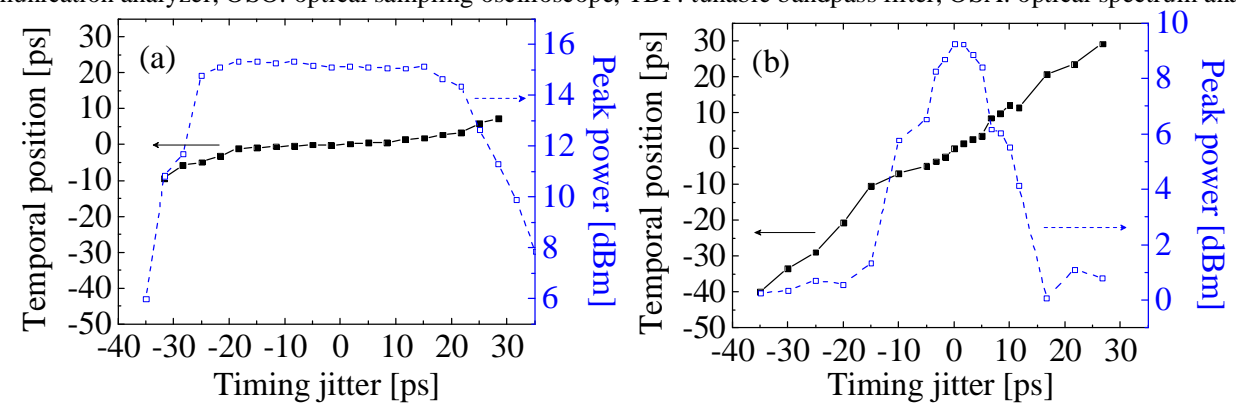

Fig. 2. Temporal position and peak power of the output signal plotted as a function of timing jitter (a) with and (b) without pre-shaping.

\section{Conclusion}

We have successfully demonstrated all-optical signal retiming based on cSHG/DFG in a fully fiberized 30-mm-long PPLN waveguide. Error-free operation was obtained for all channels with a power penalty below $0.6 \mathrm{~dB}$.

\section{Acknowledgement}

This work was supported by the UK EPSRC under grant agreement EP/F032218/1. Katia Gallo gratefully acknowledges support from the EU under grant agreement PIEF-GA-2009-234798.

\section{References}

[1] L. A. Jiang, M. E. Grein, H. A. Haus, and E. P. Ippen, “Timing jitter eater for optical pulse trains,” Opt. Lett. 28, 78-80 (2003).

[2] F. Parmigiani, P. Petropoulos, M. Ibsen, and D. J. Richardson, "All-optical pulse reshaping and retiming systems incorporating pulse shaping fiber Bragg grating," J. Lightwave Technol. 24, 357-364 (2006).

[3] K. Gallo, J. Prawiharjo, F. Parmigiani, P. Almeida, P. Petropoulos, and D. Richardson, "Processing ultrafast optical signals in broadband telecom systems by means of cascaded quadratic nonlinearities," Proc. 8th International Conference on Transparent Optical Networks (ICTON), Nottingham, UK, 18-22 Jun. 2006.

[4] T. Hasegawa, X. Wang, and A. Suzuki, "Retiming of picosecond pulses by a cascaded second-order nonlinear process in quasi-phasematched $\mathrm{LiNbO}_{3}$ waveguides," Opt. Lett. 29, 2776-2778 (2004).

[5] C. Langrock, S. Kumar, J. E. McGeehan, A. E. Willner, and M. M. Fejer, "All-optical signal processing using $\chi^{(2)}$ nonlinearities in guidedwave devices," J. Lightwave Technol. 24, 2579-2592 (2006). 